\title{
Integral Representations for the Solutions of the Generalized Schroedinger Equation in a Finite Interval
}

\author{
Anar Adiloglu Nabiev, Rauf Kh. Amirov \\ Department of Mathematics, Cumhuriyet University, Sivas, Turkey \\ Email: aadiloglu@cumhuriyet.edu.tr, emirov@cumhuriyet.edu.tr
}

Received 9 July 2015; accepted 14 November 2015; published 17 November 2015

Copyright (C) 2015 by authors and Scientific Research Publishing Inc.

This work is licensed under the Creative Commons Attribution International License (CC BY).

http://creativecommons.org/licenses/by/4.0/

(c) (i) Open Access

\section{Abstract}

We reduce the initial value problem for the generalized Schroedinger equation with piecewiseconstant leading coefficient to the system of Volterra type integral equations and construct new useful integral representations for the fundamental solutions of the Schroedinger equation. We also investigate some significant properties of the kernels of these integral representations. The integral representations of fundamental solutions enable to obtain the basic integral equations, which are a powerful tool for solving inverse spectral problems.

\section{Keywords}

One Dimensional Schroedinger Equation, Fundamental Solutions, Transformation Operator, Integral Representation, Differential Equation with Discontinues Coefficient, Kernel of an Integral Operator, Integral Equation, Method of Successive Approximations

\section{Introduction}

We consider the differential equation

$$
-y^{\prime \prime}+(q(x)+2 \lambda p(x)) y=\lambda^{2} \rho(x) y, 0 \leq x \leq \pi,
$$

where $\lambda$ is the spectral parameter, $y=y(x, \lambda)$ is an unknown function, $q(x) \in L_{2}(0, \pi), \quad p(x) \in W_{2}^{1}(0, \pi)$ are real-valued functions, and $\rho(x)$ is the following piecewise-constant function with discontinuity at the point $a \in(0, \pi)$ such that $a>\frac{\alpha \pi}{\alpha+1}$ : 


$$
\rho(x)=\left\{\begin{array}{ll}
1, & 0 \leq x \leq a \\
\alpha^{2}, & a \leq x \leq \pi
\end{array}, \quad 0<\alpha \neq 1 .\right.
$$

Sturm-Liouville equations with potentials depending on the spectral parameter arise in various fields of mathematics and physics (see [1]-[3] for details). It is well known that in the case $\rho(x)=1$ the Equation (1) appears for modelling of some problems connected with the scattering of waves and particles in physics [4]. In this classical case, Jaulent and Jean [7] [8] have constructed the integral representations of Jost solutions and treated the inverse scattering problem by the Gelfand-Levitan-Marchenko method (see [9] and [10]). Note that this method which is an effective device in the theory of inverse problems [11]-[16], for relativistic scattering problems was first suggested in [5] and [6]. Various inverse scattering problems for the Schroedinger equation with an energy dependent potential on the half line and full line were investigated in [24]-[30]. Direct and inverse spectral problems in a finite interval for the Equation (1) in the case $\rho(x)=1$ were first investigated in [17] [18]. For further discussing of the inverse spectral theory for Equation (1) in a finite interval with $\rho(x)=1$ we refer to works [19]-[23].

Note that, in the case $p(x)=0$ direct and inverse problems for boundary-value problems generated by an equation of type (1), in various formulations, have been studied in [32]-[37] and other works. Inverse scattering problem for Equation (1) with $p(x)=0$ on the half line $[0,+\infty)$ was investigated and the complete solution of this problem was given in [38] where the new integral representation, similar to transformation operators [9], was obtained for the Jost solution of the discontinuous Sturm-Liouville equation. Direct and inverse scattering problems on the half-line for the discontinuous Sturm-Liouville equation with eigenparameter dependent boundary conditions have been investigated in [41]. The direct and inverse spectral problem for the Equation (1) in the case $p(x)=0$ with some separated boundary conditions on the interval $(0, \pi)$ recently has been investigated in [39] [40] [42], where the new integral representations for solutions have been also constructed. The inverse spectral problem of recovering pencils of second-order differential operators on the half-axis with turning points was studied in [31], where the properties of spectral characteristics were established, formulation of the inverse problem was given and a uniqueness theorem for solution of the inverse problem is proven. But the spectral problems for Equation (1) in a finite interval, especially, inverse spectral problems and full-line inverse scattering problems requiring the recovery of the potential functions by the Gelfand-Levitan-Marchenko methods have not been studied yet and there isn't any serious work published in this direction.

In this work, we reduce the differential Equation (1) with initial conditions (3) to the system of Volterra type integral equations and we construct new useful integral representations for the fundamental solutions of the Equation (1). In Section 2, we consider a pair of linearly independent solutions of the Equation (1) with initial conditions at zero. We seek special Fourier-type integral forms for these solutions. To prove the existence such forms, we derive the system of Volterra type integral equations for the kernel functions. Then we solve these systems by the successive approximation method. In Section 3, we investigate some significant properties of the kernels of these integral representations. Namely, we find an important relationship between the kernels of the integrals and the coefficient of the Equation (1). The constructed integral representations of fundamental solutions play an important role in the derivation of main integral equations which are a powerful tool for solving inverse spectral problems for the Equation (1).

\section{Derivation of the Integral Representations for the Solutions}

We seek a couple of linearly independent solutions $y_{j}(x, \lambda) \quad(j=1,2)$ of Equation (1) satisfying the initial conditions

$$
y_{j}(0, \lambda)=1, y_{j}^{\prime}(0, \lambda)=(-1)^{j+1} i \lambda \text {. }
$$

İt is not difficult to show that when $q(x) \equiv p(x) \equiv 0$ the initial value problem (1), (3) has solution

where

$$
e_{j}(x, \lambda)=r^{+}(x) \mathrm{e}^{\omega_{j} \lambda \mu^{+}(x)}+r^{-}(x) \mathrm{e}^{\omega_{j} \lambda \mu^{-}(x)},
$$

$$
\mu^{ \pm}(x)= \pm x \sqrt{\rho(x)}+a(1 \mp \sqrt{\rho(x)}), r^{ \pm}(x)=\frac{1}{2}\left(1 \pm \frac{1}{\sqrt{\rho(x)}}\right)
$$


and $\omega_{j}=(-1)^{j+1} i$.

Consider the integral equation

$$
y_{j}(x, \lambda)=e_{j}(x, \lambda)+\int_{0}^{x} \Phi(x, t, \lambda)(q(t)+2 \lambda) y_{j}(t, \lambda) \mathrm{d} t(j=1 ; 2)
$$

which is equivalent to the problem (1), (3). Here

$$
\Phi(x, t, \lambda)=\frac{e_{1}(x, \lambda) e_{2}(t, \lambda)-e_{1}(t, \lambda) e_{2}(x, \lambda)}{2 i \lambda} .
$$

By using (4) it is easily obtained that

$$
\Phi(x, t, \lambda)=p^{+}(x, t) \frac{\sin \lambda \sigma^{+}(x, t)}{\lambda}-p^{-}(x, t) \frac{\sin \lambda \sigma^{-}(x, t)}{\lambda}, 0 \leq t \leq x
$$

where

We have

$$
p^{ \pm}(x, t)=\frac{1}{2}\left(\frac{1}{\sqrt{\rho(x)}} \pm \frac{1}{\sqrt{\rho(t)}}\right) \text { and } \sigma^{ \pm}(x, t)=\mu^{ \pm}(x)-\mu^{+}(t)
$$

The formula (8) is also written as

$$
\begin{aligned}
2 i \lambda \Phi(x, t, \lambda) \mathrm{e}^{\omega_{j} \lambda \mu^{ \pm}(t)}= & (-1)^{j+1} p^{ \pm}(x, t)\left[\mathrm{e}^{\omega_{j} \lambda \mu^{+}(x)}-\mathrm{e}^{\omega_{j} \lambda\left(2 \mu^{ \pm}(t)-\mu^{+}(x)\right)}\right] \\
& +(-1)^{j} p^{\mp}(x, t)\left[\mathrm{e}^{\omega_{j} \lambda \mu^{-}(x)}-\mathrm{e}^{\omega_{j} \lambda\left(2 \mu^{ \pm}(t)-\mu^{-}(x)\right)}\right] .
\end{aligned}
$$

$$
\Phi(x, t, \lambda) \mathrm{e}^{\omega_{j} \lambda \mu^{ \pm}(t)}=\frac{1}{2} p^{ \pm}(x, t) \int_{2 \mu^{ \pm}(t)-\mu^{+}(x)}^{\mu^{+}(x)} \mathrm{e}^{\omega_{j} \lambda s} \mathrm{~d} s-\frac{1}{2} p^{\mp}(x, t) \int_{2 \mu^{ \pm}(t)-\mu^{-}(x)}^{\mu^{-}(x)} \mathrm{e}^{\omega_{j} \lambda s} \mathrm{~d} s
$$

Consider the integral Equation (5) and substitute

$$
y_{j}(x, \lambda)=R_{j}^{+}(x) \mathrm{e}^{\omega_{j} \lambda \mu^{+}(x)}+R_{j}^{-}(x) \mathrm{e}^{\omega_{j} \lambda \mu^{-}(x)}+z_{j}(x, \lambda),
$$

where $R_{j}^{ \pm}(x)$ will be defined below and $z_{j}(x, \lambda)$ is a new unknown function. We have

$$
\begin{aligned}
& R_{j}^{+}(x) \mathrm{e}^{\omega_{j} \lambda \mu^{+}(x)}+R_{j}^{-}(x) \mathrm{e}^{\omega_{j} \lambda \mu^{-}(x)}+z_{j}(x, \lambda) \\
& =r^{+}(x) \mathrm{e}^{\omega_{j} \lambda \mu^{+}(x)}+r^{-}(x) \mathrm{e}^{\omega_{j} \lambda \mu^{-}(x)} \\
& +\int_{0}^{x} \Phi(x, t, \lambda)[q(t)+2 \lambda p(t)]\left[R_{j}^{+}(t) \mathrm{e}^{\omega_{j} \lambda \mu^{+}(t)}+R_{j}^{-}(t) \mathrm{e}^{\omega_{j} \lambda \mu^{-}(t)}\right] \mathrm{d} t \\
& +\int_{0}^{x} \Phi(x, t, \lambda)[q(t)+2 \lambda p(t)] z_{j}(t, \lambda) \mathrm{d} t .
\end{aligned}
$$

Taking into our account (8) and the second integral in the right hand side of (10) we require

$$
\begin{aligned}
& R_{j}^{+}(x) \mathrm{e}^{\omega_{j} \lambda \mu^{+}(x)}+R_{j}^{-}(x) \mathrm{e}^{\omega_{j} \lambda \mu^{-}(x)} \\
= & r^{+}(x) \mathrm{e}^{\omega_{j} \lambda \mu^{+}(x)}+r^{-}(x) \mathrm{e}^{\omega_{j} \lambda \mu^{-}(x)}+i(-1)^{j} \mathrm{e}^{\omega_{j} \lambda \mu^{+}(x)} \int_{0}^{x} p(t) R_{j}^{+}(t) p^{+}(x, t) \mathrm{d} t \\
& -i(-1)^{j} \mathrm{e}^{\omega_{j} \lambda \mu^{-}(x)} \int_{0}^{x} p(t) R_{j}^{+}(t) p^{-}(x, t) \mathrm{d} t+i(-1)^{j} \mathrm{e}^{\omega_{j} \lambda \mu^{+}(x)} \int_{0}^{x} p(t) R_{j}^{-}(t) p^{-}(x, t) \mathrm{d} t \\
& -i(-1)^{j} \mathrm{e}^{\omega_{j} \lambda \mu \mu^{-}(x)} \int_{0}^{x} p(t) R_{j}^{-}(t) p^{+}(x, t) \mathrm{d} t
\end{aligned}
$$


to be satisfied. Obviously, the last equality will be satisfied if we choose

$$
R_{j}^{ \pm}(x)=r^{ \pm}(x) \pm i(-1)^{j} \int_{0}^{x} p(t) R_{j}^{+}(t) p^{ \pm}(x, t) \mathrm{d} t \pm i(-1)^{j} \int_{0}^{x} p(t) R_{j}^{-}(t) p^{\mp}(x, t) \mathrm{d} t .
$$

From (11) we immediately have

$$
R_{j}^{ \pm}(x)=r^{ \pm}(x) \mathrm{e}^{\mp \omega_{j} \int_{0}^{x} \operatorname{sgn}(t \pm a) \frac{p(t)}{\sqrt{\rho(t)}} \mathrm{d} t}
$$

Then (10) implies that

$$
\begin{gathered}
z_{j}(x, \lambda)=J^{(j)}(x, \lambda)+\int_{0}^{x} \Phi(x, t, \lambda) q(t)\left[R_{j}^{+}(t) \mathrm{e}^{\omega_{j} \lambda \mu^{+}(t)}+R_{j}^{-}(t) \mathrm{e}^{\omega_{j} \lambda \mu^{-}(t)}\right] \mathrm{d} t \\
+\int_{0}^{x} \Phi(x, t, \lambda)([q(t)+2 \lambda p(t)]) z_{j}(t, \lambda) \mathrm{d} t \\
J^{(j)}(x, \lambda)=\int_{-\mu^{+}(x)}^{\mu^{+}(x)} A_{j}(x, t) \mathrm{e}^{\omega_{j} \lambda t} \mathrm{~d} t,
\end{gathered}
$$

where

and

$$
A_{j}(x, t)=\frac{\omega_{j}}{2} p\left(\frac{x+t}{2}\right) R_{j}^{+}\left(\frac{x+t}{2}\right), 0 \leq x \leq a
$$

$$
\begin{gathered}
A_{j}(x, t)=\frac{\omega_{j}}{2 \alpha^{2}} p\left(\frac{t-\mu^{-}(x)}{2 \alpha}+a\right) R_{j}^{+}\left(\frac{t-\mu^{-}(x)}{2 \alpha}\right)-\frac{\omega_{j}}{2 \alpha^{2}} p\left(\frac{\mu^{+}(x)-t}{2 \alpha}+a\right) R_{j}^{-}\left(\frac{\mu^{+}(x)-t}{2 \alpha}+a\right) \\
+\frac{\omega_{j} \alpha^{-}}{2} p\left(\frac{t+\mu^{-}(x)}{2}\right) R_{j}^{+}\left(\frac{t+\mu^{-}(x)}{2}\right), \quad \mu^{-}(x) \leq t \leq \mu^{+}(x), x>a \\
A_{j}(x, t)=\frac{\omega_{j} \alpha^{+}}{2} p\left(\frac{t+\mu^{+}(x)}{2}\right) R_{j}^{+}\left(\frac{t+\mu^{+}(x)}{2}\right)+\frac{\omega_{j} \alpha^{-}}{2} p\left(\frac{t+\mu^{-}(x)}{2}\right) R_{j}^{+}\left(\frac{t+\mu^{-}(x)}{2}\right), \\
-\mu^{-}(x)<t<\mu^{-}(x), x>a \\
A_{j}(x, t)=\frac{\omega_{j} \alpha^{+}}{2} p\left(\frac{t+\mu^{+}(x)}{2}\right) R_{j}^{+}\left(\frac{t+\mu^{+}(x)}{2}\right),-\mu^{+}(x) \leq t<-\mu^{-}(x), x>a .
\end{gathered}
$$

We require that the integral Equation (13) has the solution

$$
z_{j}(x, \lambda)=\int_{-\mu^{+}(x)}^{\mu^{+}(x)} K_{j}(x, t) \mathrm{e}^{\omega_{j} \lambda t} \mathrm{~d} t,
$$

where $K_{j}(x, t)$ is an unknown function. Substituting the expression (16) of $z_{j}(x, \lambda)$ in the Equation (13) we have

$$
\begin{aligned}
\int_{-\mu^{+}(x)}^{\mu^{+}(x)} K_{j}(x, t) \mathrm{e}^{\omega_{j} \lambda t} \mathrm{~d} t= & \int_{-\mu^{+}(x)}^{\mu^{+}(x)} A_{j}(x, t) \mathrm{e}^{\omega_{j} \lambda t} \mathrm{~d} t+\int_{0}^{x} \Phi(x, t, \lambda) \mathrm{e}^{\omega_{j} \lambda \mu^{+}(t)} q(t) R_{j}^{+}(t) \mathrm{d} t \\
& +\int_{0}^{x} \Phi(x, t, \lambda) \mathrm{e}^{\omega_{j} \lambda \mu^{-}(t)} q(t) R_{j}^{-}(t) \mathrm{d} t \\
& +\int_{0}^{x}[q(t)+2 \lambda p(t)] \int_{-\mu^{+}(t)}^{\mu^{+}(t)} K_{j}(t, s) \Phi(x, t ; \lambda) \mathrm{e}^{\omega_{j} \lambda s} \mathrm{~d} s \mathrm{~d} t
\end{aligned}
$$


Now using the formulas (8), (8') we transform the right hand side of Equation (16) to the form of the Fourier integral.

First consider the case $0 \leq x \leq a$ for which the Equation (16) is written as

$$
\begin{aligned}
\int_{-x}^{x} K_{j}(x, t) \mathrm{e}^{\omega_{j} \lambda t} \mathrm{~d} t= & \int_{-x}^{x} \frac{\omega_{j}}{2} p\left(\frac{x+t}{2}\right) R_{j}^{+}\left(\frac{x+t}{2}\right) \mathrm{e}^{\omega_{j} \lambda t} \mathrm{~d} t+\frac{1}{2} \int_{0}^{x} q(t) R_{j}^{+}(t) \mathrm{d} t \int_{2 t-x}^{x} \mathrm{e}^{\omega_{j} \lambda \xi} \mathrm{d} \xi \\
& +\frac{1}{2} \int_{0}^{x} q(t) \int_{-t}^{t} K_{j}(t, s) \int_{s-x+t}^{s+x-t} \mathrm{e}^{\omega_{j} \lambda \xi} \mathrm{d} \xi \mathrm{d} s \mathrm{~d} t \\
& -\omega_{j} \int_{0}^{x}\left[\mathrm{e}^{\omega_{j} \lambda(x-t)}-\mathrm{e}^{-\omega_{j} \lambda(x-t)}\right] p(t) \int_{-t}^{t} K_{j}(t, s) \mathrm{e}^{\omega_{j} \lambda s} \mathrm{~d} s \mathrm{~d} t .
\end{aligned}
$$

Supposing $K_{j}(x, t)$ to be zero as $|t|>x$ and changing orders of integrations at the right hand side of Equation (17) we obtain

$$
\begin{aligned}
& \int_{-x}^{x} K_{j}(x, t) \mathrm{e}^{\omega_{j} \lambda t} \mathrm{~d} t \int_{-x}^{x} \mathrm{e}^{\omega_{j} \lambda t}\left\{\frac{1}{2} \int_{0}^{\frac{x+t}{2}} q(s) R_{j}^{+}(s) \mathrm{d} s+\frac{\omega_{j}}{2} p\left(\frac{x+t}{2}\right) R_{j}^{+}\left(\frac{x+t}{2}\right) \mathrm{d} t+\frac{1}{2} \int_{0}^{x} q(s) \mathrm{d} s \int_{t-x+s}^{t+x-s} K_{j}(s, \xi) \mathrm{d} \xi\right. \\
& \left.-\omega_{j}\left[\int_{\frac{x-t}{2}}^{x} p(s) K_{j}(s, t-x+s) \mathrm{d} s-\int_{\frac{x+t}{2}}^{x} p(s) K_{j}(s, t+x-s) \mathrm{d} s\right]\right\}
\end{aligned}
$$

According to the uniqueness properties of the Fourier transformation, Equation (18) implies that

$$
\begin{aligned}
K_{j}(x, t)= & \left\{\frac{1}{2} \int_{0}^{\frac{x+t}{2}} q(s) R_{j}^{+}(s) \mathrm{d} s+\frac{\omega_{j}}{2} p\left(\frac{x+t}{2}\right) R_{j}^{+}\left(\frac{x+t}{2}\right) \mathrm{d} t+\frac{1}{2} \int_{0}^{x} q(s) \mathrm{d} s \int_{t-x+s}^{t+x-s} K_{j}(s, \xi) \mathrm{d} \xi\right. \\
& \left.-\omega_{j}\left[\int_{\frac{x-t}{2}}^{x} p(s) K_{j}(s, t-x+s) \mathrm{d} s-\int_{\frac{x+t}{2}}^{x} p(s) K_{j}(s, t+x-s) \mathrm{d} s\right]\right\},|t| \leq x .
\end{aligned}
$$

Now consider the case $x>a$. In this case, according to formulas (8) and (8'), the Equation (16) yields

$$
\begin{aligned}
& \int_{-\mu^{+}(x)}^{\mu^{+}(x)} K_{j}(x, t) \mathrm{e}^{\omega_{j} \lambda^{n} t} \mathrm{~d} t=\int_{-\mu^{+}(x)}^{\mu^{+}(x)} A_{j}(x, t) \mathrm{e}^{\omega_{j} \lambda t} \mathrm{~d} t+\frac{1}{2} \int_{0}^{a} q(t) R_{j}^{+}(t) \mathrm{d} t\left[\alpha^{+} \int_{2 t-\mu^{+}(x)}^{\mu^{+}(x)} \mathrm{e}^{\omega_{j} \lambda s} \mathrm{~d} s+\alpha^{-} \int_{2 t-\mu^{-}(x)}^{\mu^{-}(x)} \mathrm{e}^{\omega_{j} \lambda s} \mathrm{~d} s\right] \\
& +\int_{a}^{x} \frac{1}{2 \alpha} q(t)\left[R_{j}^{+}(t) \int_{2 \mu^{+}(t)-\mu^{+}(x)}^{\mu^{+}(x)} \mathrm{e}^{\omega_{j} \lambda s} \mathrm{~d} s+R_{j}^{-}(t) \int_{\mu^{-}(x)}^{2 \mu^{-}(t)-\mu^{-}(x)} \mathrm{e}^{\omega_{j} \lambda s} \mathrm{~d} s\right] \mathrm{d} t \\
& +\frac{1}{2} \int_{0}^{a} q(t) \mathrm{d} t \int_{-t}^{t} K_{j}(t, s) \mathrm{d} s\left[\alpha^{+} \int_{t-\mu^{+}(x)+s}^{\mu^{+}(x)-t+s} \mathrm{e}^{\omega_{j} \lambda \xi} \mathrm{d} \xi+\alpha^{-} \int_{t-\mu^{-}(x)+s}^{\mu^{-}(x)-t+s} \mathrm{e}^{\omega_{j} \lambda \xi} \mathrm{d} \xi\right] \\
& -\alpha^{+} \omega_{j} \int_{0}^{a} p(t) \mathrm{d} t \int_{-t}^{t} K_{j}(t, s)\left[\mathrm{e}^{\omega_{j} \lambda\left(s+\mu^{+}(x)-t\right)}-\mathrm{e}^{\omega_{j} \lambda\left(s-\mu^{+}(x)+t\right)}\right] \mathrm{d} s \\
& -\alpha^{-} \omega_{j} \int_{0}^{a} p(t) \mathrm{d} t \int_{-t}^{t} K_{j}(t, s)\left[\mathrm{e}^{\omega_{j} \lambda\left(s+\mu^{-}(x)-t\right)}-\mathrm{e}^{\omega_{j} \lambda\left(s-\mu^{-}(x)+t\right)}\right] \mathrm{d} s \\
& +\int_{a}^{x} \frac{1}{2 \alpha} q(t) \mathrm{d} t \int_{-\mu^{+}(t)}^{\mu^{+}(t)} K_{j}(t, s) \mathrm{d} s \int_{s+\mu^{+}(t)-\mu^{+}(x)}^{s+\mu^{+}(x)-\mu^{+}(t)} \mathrm{e}^{\omega_{j} \lambda \xi} \mathrm{d} \xi \\
& -\frac{\omega_{j}}{\alpha} \int_{a}^{x} p(t) \mathrm{d} t \int_{-\mu^{+}(t)}^{\mu^{+}(t)} K_{j}(t, s)\left[\mathrm{e}^{\omega_{j} \lambda\left(s+\mu^{+}(x)-\mu^{+}(t)\right)}-\mathrm{e}^{\omega_{j} \lambda\left(s-\mu^{+}(x)+\mu^{+}(t)\right)}\right] \mathrm{d} s .
\end{aligned}
$$


Now, similar to previous case we obtain from the Equation (20) that the function $K_{j}(x, t)(x>a)$, continued as zero for $|t|>\boldsymbol{\mu}^{+}(x)$, satisfies some integral equations of type (19) in the corresponding regions. Namely we have the following:

1) if $-\boldsymbol{\mu}^{+}(x) \leq \boldsymbol{t}<-\boldsymbol{\mu}^{-}(x)$ then

$$
\begin{aligned}
K_{j}(x, t)= & \frac{\alpha^{+}}{2} \int_{0}^{\frac{\mu^{+}(x)+t}{2}} q(s) R_{j}^{+}(s) \mathrm{d} s+\frac{\omega_{j} \alpha^{+}}{2} p\left(\frac{t+\mu^{+}(x)}{2}\right) R_{j}^{+}\left(\frac{t+\mu^{+}(x)}{2}\right) \\
& +\frac{\alpha^{+}}{2} \int_{0}^{a} q(s) \mathrm{d} s \int_{-s}^{\min \left(s, t+\mu^{+}(x)-s\right)} K_{j}(s, \xi) \mathrm{d} \xi-\frac{\alpha^{-}}{2} \int_{\frac{\mu^{-}(x)-t}{2}}^{a} q(s) \mathrm{d} s \int_{-s}^{t-\mu^{-}(x)+s} K_{j}(s, \xi) \mathrm{d} \xi \\
& +\frac{1}{2 \alpha} \int_{a}^{x} q(s) \mathrm{d} s \int_{-\mu^{+}(s)}^{t+\mu^{+}(x)-\mu^{+}(s)} K_{j}(s, \xi) \mathrm{d} \xi-\frac{1}{2 \alpha} \int_{a-\frac{\mu^{-}(x)+t}{2 \alpha}}^{x} q(s) \mathrm{d} s \int_{-\mu^{+}(s)}^{t-\mu^{+}(x)+\mu^{+}(s)} K_{j}(s, \xi) \mathrm{d} \xi \\
& +\omega_{j} \alpha^{+} \int_{\frac{1}{t+\mu^{+}(x)}}^{2} p(s) K_{j}\left(s, t+\mu^{+}(x)-s\right) \mathrm{d} s-\omega_{j} \alpha^{-} \int_{\frac{\mu^{-}(x)-t}{2}}^{a} p(s) K_{j}\left(s, t-\mu^{-}(x)+s\right) \mathrm{d} s \\
& -\frac{\omega_{j}}{\alpha} \int_{a-\frac{\mu^{-}(x)+t}{2 \alpha}}^{x} p(s) K_{j}\left(s, t-\mu^{+}(x)+\mu^{+}(s)\right) \mathrm{d} s+\frac{\omega_{j}}{\alpha} \int_{a}^{x} p(s) K_{j}\left(s, t+\mu^{+}(x)-\mu^{+}(s)\right) \mathrm{d} s .
\end{aligned}
$$

2) if $-\boldsymbol{\mu}^{-}(x) \leq \boldsymbol{t}<\boldsymbol{\mu}^{-}(x)$ then

$$
\begin{aligned}
K_{j}(x, t)= & \frac{\alpha^{+}}{2} \int_{0}^{\frac{\mu^{+}(x)+t}{2}} q(s) R_{j}^{+}(s) \mathrm{d} s+\frac{\alpha^{-}}{2} \int_{0}^{\frac{\mu^{-}(x)+t}{2}} q(s) R_{j}^{+}(s) \mathrm{d} s \\
& +\frac{\omega_{j} \alpha^{+}}{2} p\left(\frac{t+\mu^{+}(x)}{2}\right) R_{j}^{+}\left(\frac{t+\mu^{+}(x)}{2}\right)+\frac{\omega_{j} \alpha^{-}}{2} p\left(\frac{t+\mu^{-}(x)}{2}\right) R_{j}^{+}\left(\frac{t+\mu^{-}(x)}{2}\right) \\
& +\frac{\alpha^{+}}{2} \int_{0}^{a} q(s) \mathrm{d} s \int_{-s}^{\min \left(s, t+\mu^{+}(x)-s\right)} K_{j}(s, \xi) \mathrm{d} \xi-\frac{\alpha^{+}}{2} \int_{\frac{\mu^{+}(x)-t}{2}}^{a} q(s) \mathrm{d} s \int_{-s}^{t-\mu^{+}(x)+s} K_{j}(s, \xi) \mathrm{d} \xi \\
& -\frac{\alpha^{-}}{2} \int_{\frac{\mu^{-}(x)-t}{2}}^{a} q(s) \mathrm{d} s \int_{-s}^{t-\mu^{-}(x)+s} K_{j}(s, \xi) \mathrm{d} \xi+\frac{\alpha^{-}}{2} \int_{0}^{a} q(s) \mathrm{d} s \int_{-s}^{\min \left(s, t+\mu^{-}(x)-s\right)} K_{j}(s, \xi) \mathrm{d} \xi \\
& +\frac{1}{2 \alpha} \int_{a}^{x} q(s) \mathrm{d} s \int_{t-\mu^{+}(x)+\mu^{+}(s)}^{t+\mu^{+}(x)-\mu^{+}(s)} K_{j}(s, \xi) \mathrm{d} \xi+\omega_{j} \alpha^{+} \int_{\frac{\mu^{+}(x)+t}{2}}^{a} p(s) K_{j}\left(s, t+\mu^{+}(x)-s\right) \mathrm{d} s \\
& -\omega_{j} \alpha^{+} \int_{\frac{\mu^{+}(x)-t}{2}}^{a} p(s) K_{j}\left(s, t-\mu^{+}(x)+s\right) \mathrm{d} s+\omega_{j} \alpha^{-} \int_{\mu^{-}(x)+t}^{a} p(s) K_{j}\left(s, t+\mu^{-}(x)-s\right) \mathrm{d} s \\
& -\omega_{j} \alpha^{-} \int_{\frac{\mu^{-}(x)-t}{2}}^{a} p(s) K_{j}\left(s, t-\mu^{-}(x)+s\right) \mathrm{d} s-\frac{\omega_{j}}{\alpha} \int_{a}^{x} p(s) K_{j}\left(s, t-\mu^{+}(x)+\mu^{+}(s)\right) \mathrm{d} s \\
& +\frac{\omega_{j}}{\alpha} \int_{a}^{x} p(s) K_{j}\left(s, t+\mu^{+}(x)-\mu^{+}(s)\right) \mathrm{d} s .
\end{aligned}
$$

3) if $\boldsymbol{\mu}^{-}(x) \leq \boldsymbol{t}<\boldsymbol{\mu}^{+}(x)$ then 


$$
\begin{aligned}
& K_{j}(x, t)=\frac{\alpha^{+}}{2} \int_{0}^{a} q(s) R_{j}^{+}(s) \mathrm{d} s-\frac{\alpha^{-}}{2} \int_{\frac{\mu^{-}(x)+t}{2}}^{a} q(s) R_{j}^{+}(s) \mathrm{d} s+\frac{1}{2 \alpha} \int_{a}^{a+\frac{t-\mu^{-}(x)}{2 \alpha}} q(s) R_{j}^{+}(s) \mathrm{d} s \\
& +\frac{1}{2 \alpha} \int_{a}^{\frac{\mu^{+}(x)-t}{2 \alpha}+a} q(s) R_{j}^{-}(s) \mathrm{d} s+\frac{\omega_{j}}{2 \alpha^{2}} p\left(\frac{t-\mu^{-}(x)}{2 \alpha}+a\right) R_{j}^{+}\left(\frac{t-\mu^{-}(x)}{2 \alpha}+a\right) \\
& -\frac{\omega_{j}}{2 \alpha^{2}} p\left(\frac{\mu^{+}(x)-t}{2 \alpha}+a\right) R_{j}^{-}\left(\frac{\mu^{+}(x)-t}{2 \alpha}+a\right) \\
& +\frac{\omega_{j} \alpha^{-}}{2} p\left(\frac{t+\mu^{-}(x)}{2}\right) R_{j}^{+}\left(\frac{t+\mu^{-}(x)}{2}\right)+\frac{\alpha^{+}}{2} \int_{0}^{a} q(s) \mathrm{d} s \int_{-s}^{s} K_{j}(s, \xi) \mathrm{d} \xi \\
& -\frac{\alpha^{+}}{2} \int_{\frac{\mu^{+}(x)-t}{2}}^{a} q(s) \mathrm{d} s \int_{-s}^{t-\mu^{+}(x)+s} K_{j}(s, \xi) \mathrm{d} \xi-\frac{\alpha^{-}}{2} \int_{0}^{a} q(s) \mathrm{d} s \int_{-s}^{s} K_{j}(s, \xi) \mathrm{d} \xi \\
& +\frac{\alpha^{-}}{2} \int_{0}^{a} q(s) \mathrm{d} s \int_{-s}^{\min \left(s, t+\mu^{-}(x)-s\right)} K_{j}(s, \xi) \mathrm{d} \xi+\frac{1}{2 \alpha} \int_{a}^{x} q(s) \mathrm{d} s \int_{-\mu^{+}(s)}^{\min \left(\mu^{+}(s), t+\mu^{+}(x)-\mu^{+}(s)\right)} K_{j}(s, \xi) \mathrm{d} \xi \\
& -\frac{1}{2 \alpha} \int_{a}^{x} q(s) \mathrm{d} s \int_{-\mu^{+}(s)}^{t-\mu^{+}(x)+\mu^{+}(s)} K_{j}(s, \xi) \mathrm{d} \xi-\omega_{j} \alpha^{+} \int_{\frac{\mu^{+}(x)-t}{2}}^{a} p(s) K_{j}\left(s, t-\mu^{+}(x)+s\right) \mathrm{d} s \\
& +\omega_{j} \alpha^{-} \int_{\frac{\mu^{-}(x)+t}{2}}^{a} p(s) K_{j}\left(s, t+\mu^{-}(x)-s\right) \mathrm{d} s-\frac{\omega_{j}}{\alpha} \int_{a}^{x} p(s) K_{j}\left(s, t-\mu^{+}(x)+\mu^{+}(s)\right) \mathrm{d} s \\
& +\frac{\omega_{j}}{\alpha} \int_{a+\frac{t-\mu^{-}(x)}{2 \alpha}}^{x} p(s) K_{j}\left(s, t+\mu^{+}(x)-\mu^{+}(s)\right) \mathrm{d} s .
\end{aligned}
$$

Now we use the method of the successive approximation to show that for every fixed $x \in[0, \pi]$ the integral Equation (19), (21)-(23) has a unique solution $K_{j}(x, t)$ belonging to $L_{1}\left(-\mu^{+}(x), \mu^{+}(x)\right)$. For this reason let us define

$$
\begin{gathered}
K_{j}^{(0)}(x, t)=\frac{1}{2} \int_{0}^{\frac{x+t}{2}} q(s) R_{j}^{+}(s) \mathrm{d} s+\frac{\omega_{j}}{2} p\left(\frac{x+t}{2}\right) R_{j}^{+}\left(\frac{x+t}{2}\right) \mathrm{d} t, \\
K_{j}^{(n)}(x, t)=\frac{1}{2} \int_{0}^{x} q(s) \mathrm{d} s \int_{-s}^{\min (s, t+x-s)} K_{j}^{(n-1)}(s, \xi) \mathrm{d} \xi-\frac{1}{2} \int_{\frac{x-t}{2}}^{x} q(s) \mathrm{d} s \int_{-s}^{t-x+s} K_{j}^{(n-1)}(s, \xi) \mathrm{d} \xi \\
-\omega_{j}\left[\int_{\frac{x-t}{2}}^{x} p(s) K_{j}^{(n-1)}(s, t-x+s) \mathrm{d} s-\int_{\frac{x+t}{2}}^{x} p(s) K_{j}^{(n-1)}(s, t+x-s) \mathrm{d} s\right], \\
|t| \leq x \leq a, n=1,2, \cdots, \\
K_{j}^{(0)}(x, t)=\frac{\alpha^{+}}{2} \int_{0}^{\frac{\mu^{+}(x)+t}{2}} q(s) R_{j}^{+}(s) \mathrm{d} s+\frac{\omega_{j} \alpha^{+}}{2} p\left(\frac{t+\mu^{+}(x)}{2}\right) R_{j}^{+}\left(\frac{t+\mu^{+}(x)}{2}\right),
\end{gathered}
$$




$$
\begin{aligned}
& K_{j}^{(n)}(x, t)=\frac{\alpha^{+}}{2} \int_{0}^{a} q(s) \mathrm{d} s \int_{-s}^{\min \left(s, t+\mu^{+}(x)-s\right)} K_{j}^{(n-1)}(s, \xi) \mathrm{d} \xi-\frac{\alpha^{-}}{2} \int_{\frac{\mu^{-}(x)-t}{2}}^{a} q(s) \mathrm{d} s \int_{-s}^{t-\mu^{-}(x)+s} K_{j}^{(n-1)}(s, \xi) \mathrm{d} \xi \\
& +\frac{1}{2 \alpha} \int_{a}^{x} q(s) \mathrm{d} s \int_{-\mu^{+}(s)}^{t+\mu^{+}(x)-\mu^{+}(s)} K_{j}^{(n-1)}(s, \xi) \mathrm{d} \xi-\frac{1}{2 \alpha} \int_{a-\frac{\mu^{-}(x)+t}{2 \alpha}}^{x} q(s) \mathrm{d} s \int_{-\mu^{+}(s)}^{t-\mu^{+}(x)+\mu^{+}(s)} K_{j}^{(n-1)}(s, \xi) \mathrm{d} \xi \\
& +\omega_{j} \alpha^{+} \int_{\frac{t+\mu^{+}(x)}{2}}^{a} p(s) K_{j}^{(n-1)}\left(s, t+\mu^{+}(x)-s\right) \mathrm{d} s-\omega_{j} \alpha^{-} \int_{\frac{\mu^{-}(x)-t}{2}}^{a} p(s) K_{j}^{(n-1)}\left(s, t-\mu^{-}(x)+s\right) \mathrm{d} s \\
& -\frac{\omega_{j}}{\alpha} \int_{a-\frac{\mu^{-}(x)+t}{2 \alpha}}^{x} p(s) K_{j}^{(n-1)}\left(s, t-\mu^{+}(x)+\mu^{+}(s)\right) \mathrm{d} s \\
& +\frac{\omega_{j}}{\alpha} \int_{a}^{x} p(s) K_{j}^{(n-1)}\left(s, t+\mu^{+}(x)-\mu^{+}(s)\right) \mathrm{d} s, \\
& x>a,-\mu^{+}(x) \leq t<-\mu^{-}(x), n=1,2, \cdots \\
& K_{j}^{(0)}(x, t)=\frac{\alpha^{+}}{2} \int_{0}^{\frac{\mu^{+}(x)+t}{2}} q(s) R_{j}^{+}(s) \mathrm{d} s+\frac{\alpha^{-}}{2} \int_{0}^{\frac{\mu^{-}(x)+t}{2}} q(s) R_{j}^{+}(s) \mathrm{d} s \\
& +\frac{\omega_{j} \alpha^{+}}{2} p\left(\frac{t+\mu^{+}(x)}{2}\right) R_{j}^{+}\left(\frac{t+\mu^{+}(x)}{2}\right) \\
& +\frac{\omega_{j} \alpha^{-}}{2} p\left(\frac{t+\mu^{-}(x)}{2}\right) R_{j}^{+}\left(\frac{t+\mu^{-}(x)}{2}\right) \text {, } \\
& K_{j}^{(n)}(x, t)=\frac{\alpha^{+}}{2} \int_{0}^{a} q(s) \mathrm{d} s \int_{-s}^{\min \left(s, t+\mu^{+}(x)-s\right)} K_{j}^{(n-1)}(s, \xi) \mathrm{d} \xi-\frac{\alpha^{+}}{2} \int_{\frac{\mu^{+}(x)-t}{2}}^{a} q(s) \mathrm{d} s \int_{-s}^{t-\mu^{+}(x)+s} K_{j}^{(n-1)}(s, \xi) \mathrm{d} \xi \\
& -\frac{\alpha^{-}}{2} \int_{\frac{\mu^{-}(x)-t}{2}}^{a} q(s) \mathrm{d} s \int_{-s}^{t-\mu^{-}(x)+s} K_{j}^{(n-1)}(s, \xi) \mathrm{d} \xi+\frac{\alpha^{-}}{2} \int_{0}^{a} q(s) \mathrm{d} s \int_{-s}^{\min \left(s, t+\mu^{-}(x)-s\right)} K_{j}^{(n-1)}(s, \xi) \mathrm{d} \xi \\
& +\frac{1}{2 \alpha} \int_{a}^{x} q(s) \mathrm{d} s \int_{t-\mu^{+}(x)+\mu^{+}(s)}^{t+\mu^{+}(x)-\mu^{+}(s)} K_{j}^{(n-1)}(s, \xi) \mathrm{d} \xi+\omega_{j} \alpha^{+} \int_{\frac{\mu^{+}(x)+t}{2}}^{a} p(s) K_{j}^{(n-1)}\left(s, t+\mu^{+}(x)-s\right) \mathrm{d} s \\
& -\omega_{j} \alpha^{+} \int_{\frac{\mu^{+}(x)-t}{2}}^{a} p(s) K_{j}^{(n-1)}\left(s, t-\mu^{+}(x)+s\right) \mathrm{d} s+\omega_{j} \alpha^{-} \int_{\frac{\mu^{-}(x)+t}{2}}^{a} p(s) K_{j}^{(n-1)}\left(s, t+\mu^{-}(x)-s\right) \mathrm{d} s \\
& -\omega_{j} \alpha^{-} \int_{\frac{\mu^{-}(x)-t}{2}}^{a} p(s) K_{j}^{(n-1)}\left(s, t-\mu^{-}(x)+s\right) \mathrm{d} s-\frac{\omega_{j}}{\alpha} \int_{a}^{x} p(s) K_{j}^{(n-1)}\left(s, t-\mu^{+}(x)+\mu^{+}(s)\right) \mathrm{d} s \\
& +\frac{\omega_{j}}{\alpha} \int_{a}^{x} p(s) K_{j}^{(n-1)}\left(s, t+\mu^{+}(x)-\mu^{+}(s)\right) \mathrm{d} s, \\
& x>a,-\boldsymbol{\mu}^{-}(x) \leq \boldsymbol{t}<\boldsymbol{\mu}^{-}(x), n=1,2, \cdots
\end{aligned}
$$




$$
\begin{aligned}
& K_{j}^{(0)}(x, t)=\frac{\alpha^{+}}{2} \int_{0}^{a} q(s) R_{j}^{+}(s) \mathrm{d} s-\frac{\alpha^{-}}{2} \int_{\frac{\mu^{-}(x)+t}{2}}^{a} q(s) R_{j}^{+}(s) \mathrm{d} s+\frac{1}{2 \alpha} \int_{a}^{a+\frac{t-\mu^{-}(x)}{2 \alpha}} q(s) R_{j}^{+}(s) \mathrm{d} s \\
& +\frac{1}{2 \alpha} \int_{a}^{\frac{\mu^{+}(x)-t}{2 \alpha}+a} q(s) R_{j}^{-}(s) \mathrm{d} s+\frac{\omega_{j}}{2 \alpha^{2}} p\left(\frac{t-\mu^{-}(x)}{2 \alpha}+a\right) R_{j}^{+}\left(\frac{t-\mu^{-}(x)}{2 \alpha}+a\right) \\
& -\frac{\omega_{j}}{2 \alpha^{2}} p\left(\frac{\mu^{+}(x)-t}{2 \alpha}+a\right) R_{j}^{-}\left(\frac{\mu^{+}(x)-t}{2 \alpha}+a\right)+\frac{\omega_{j} \alpha^{-}}{2} p\left(\frac{t+\mu^{-}(x)}{2}\right) R_{j}^{+}\left(\frac{t+\mu^{-}(x)}{2}\right), \\
& K_{j}^{(n)}(x, t)=\frac{\alpha^{+}}{2} \int_{0}^{a} q(s) \mathrm{d} s_{-s}^{s} K_{j}^{(n-1)}(s, \xi) \mathrm{d} \xi-\frac{\alpha^{+}}{2} \int_{\frac{\mu^{+}(x)-t}{2}}^{a} q(s) \mathrm{d} s \int_{-s}^{t-\mu^{+}(x)+s} K_{j}^{(n-1)}(s, \xi) \mathrm{d} \xi \\
& -\frac{\alpha^{-}}{2} \int_{0}^{a} q(s) \mathrm{d} s \int_{-s}^{s} K_{j}^{(n-1)}(s, \xi) \mathrm{d} \xi+\frac{\alpha^{-}}{2} \int_{0}^{a} q(s) \mathrm{d} s \int_{-s}^{\min \left(s, t+\mu^{-}(x)-s\right)} K_{j}^{(n-1)}(s, \xi) \mathrm{d} \xi \\
& +\frac{1}{2 \alpha} \int_{a}^{x} q(s) \mathrm{d} s \int_{-\mu^{+}(s)}^{\min \left(\mu^{+}(s), t+\mu^{+}(x)-\mu^{+}(s)\right)} K_{j}^{(n-1)}(s, \xi) \mathrm{d} \xi-\frac{1}{2 \alpha} \int_{a}^{x} q(s) \mathrm{d} s \int_{-\mu^{+}(s)}^{t-\mu^{+}(x)+\mu^{+}(s)} K_{j}^{(n-1)}(s, \xi) \mathrm{d} \xi \\
& -\omega_{j} \alpha^{+} \int_{\frac{\mu^{+}(x)-t}{2}}^{a} p(s) K_{j}^{(n-1)}\left(s, t-\mu^{+}(x)+s\right) \mathrm{d} s+\omega_{j} \alpha^{-} \int_{\frac{\mu^{-}(x)+t}{2}}^{a} p(s) K_{j}^{(n-1)}\left(s, t+\mu^{-}(x)-s\right) \mathrm{d} s \\
& -\frac{\omega_{j}}{\alpha} \int_{a}^{x} p(s) K_{j}^{(n-1)}\left(s, t-\mu^{+}(x)+\mu^{+}(s)\right) \mathrm{d} s+\frac{\omega_{j}}{\alpha} \int_{a+\frac{t \mu^{-}(x)}{2 \alpha}}^{x} p(s) K_{j}^{(n-1)}\left(s, t+\mu^{+}(x)-\mu^{+}(s)\right) \mathrm{d} s, \\
& x>a, \boldsymbol{\mu}^{-}(x) \leq \boldsymbol{t}<\boldsymbol{\mu}^{+}(x), n=1,2, \cdots
\end{aligned}
$$

We have

$$
\begin{gathered}
\int_{-\mu^{+}(x)}^{\mu^{+}(x)}\left|K_{j}^{(0)}(x, t)\right| \mathrm{d} t \leq \sigma(x), \\
\sigma(x)=2 C_{0} \int_{0}^{x}\left[\left(\mu^{+}(x)-\mu^{+}(s)\right)|q(s)|+|p(s)|\right],
\end{gathered}
$$

where $C_{0}=\max \left(1,\left(\alpha^{+}+\left|\alpha^{-}\right|\right)\right.$. Similarly we obtain that

$$
\begin{aligned}
\int_{-\mu^{+}(x)}^{\mu^{+}(x)}\left|K_{j}^{(n)}(x, t)\right| \mathrm{d} t \leq & \alpha^{+} \int_{0}^{a}\left(\mu^{+}(x)-s\right)|q(s)| \mathrm{d} s \int_{-s}^{s}\left|K_{j}^{(n-1)}(s, \xi)\right| \mathrm{d} \xi \\
& +\left|\alpha^{-}\right| \int_{0}^{a}\left|\mu^{-}(x)-s\right||q(s)| \mathrm{d} s \int_{-s}^{s}\left|K_{j}^{(n-1)}(s, \xi)\right| \mathrm{d} \xi+2\left(\left(\alpha^{+}+\left|\alpha^{-}\right|\right) \iint_{0}^{a}|p(s)| \mathrm{d} s\right) \int_{-s}^{s}\left|K_{j}^{(n-1)}(s, \xi)\right| \mathrm{d} \xi \\
& +\frac{1}{\alpha} \int_{a}^{x}\left(\mu^{+}(x)-\mu^{+}(s)\right)|q(s)| \mathrm{d} s \int_{-\mu^{+}(s)}^{\mu^{+}(s)}\left|K_{j}^{(n-1)}(s, \xi)\right| \mathrm{d} \xi+\frac{2}{\alpha} \int_{a}^{x}|p(s)| \mathrm{d} s \int_{-\mu^{+}(s)}^{\mu^{+}(s)}\left|K_{j}^{(n-1)}(s, \xi)\right| \mathrm{d} \xi \\
& \leq 2 C_{0} \int_{0}^{x}\left[\left(\mu^{+}(x)-\mu^{+}(s)\right)|q(s)|+|p(s)|\right] \mathrm{d} s \int_{-\mu^{+}(s)}^{\mu^{+}(s)}\left|K_{j}^{(n-1)}(s, \xi)\right| \mathrm{d} \xi, a<x \leq \pi,
\end{aligned}
$$

that is 


$$
\int_{-\mu^{+}(x)}^{\mu^{+}(x)}\left|K_{j}^{(n)}(x, t)\right| \mathrm{d} t \leq 2 C_{0} \int_{0}^{x}\left[\left(\mu^{+}(x)-\mu^{+}(s)\right)|q(s)|+|p(s)|\right] \mathrm{d} s \int_{-\mu^{+}(x)}^{\mu^{+}(x)}\left|K_{j}^{(n-1)}(s, \xi)\right| \mathrm{d} \xi
$$

for all $x \in[0, \pi]$. Therefore

$$
\int_{-\mu^{+}(x)}^{\mu^{+}(x)}\left|K_{j}^{(n)}(x, t)\right| \mathrm{d} t \leq \frac{[\sigma(x)]^{n+1}}{(n+1) !}
$$

for all $x \in[0, \pi]$ and $n=0,1,2, \cdots$. Hence the series

$$
\sum_{n=0}^{\infty} K_{j}^{(n)}(x, .)
$$

absolutely and uniformly converges in the space $L_{1}\left(-\mu^{+}(x), \mu^{+}(x)\right)$ for each $x \in[0, \pi]$, the sum $K_{j}(x,$. of this series is a unique solution of the integral Equations (19), (21)-(23) and $K_{j}(x,$.$) satisfies the inequality$

$$
\int_{-\mu^{+}(x)}^{\mu^{+}(x)}\left|K_{j}(x, t)\right| \mathrm{d} t \leq \mathrm{e}^{\sigma(x)}-1 .
$$

Therefore we have proved the following theorem:

Theorem 1. For every $\lambda$ the solution $y_{j}(x, \lambda)$ of Equation (1) satisfying the initial conditions (3) can be represented as

$$
y_{j}(x, \lambda)=R_{j}^{+}(x) \mathrm{e}^{\omega_{j} \lambda \mu^{+}(x)}+R_{j}^{-}(x) \mathrm{e}^{\omega_{j} \lambda \mu^{-}(x)}+\int_{-\mu^{+}(x)}^{\mu^{+}(x)} K_{j}(x, t) \mathrm{e}^{\omega_{j} \lambda t} \mathrm{~d} t,
$$

where

and the kernel $K_{j}(x, t)$ satisfies (33).

$$
R_{j}^{ \pm}(x)=r^{ \pm}(x) \mathrm{e}^{\mp \omega_{j} j \operatorname{sgn}(t \pm a)} \frac{p(t)}{\sqrt{\rho(t)}} \mathrm{dt}
$$

\section{Properties of the Kernels}

From the integral Equations (19), (21)-(23) we easily compute the following boundary relations for $K_{j}(x,$.$) :$

1) if $0 \leq x \leq a$ then from Equation (19) we have

$$
K_{j}(x,-x)=\frac{\omega_{j}}{2} p(0)+\omega_{j} \int_{0}^{x} p(s) K_{j}(s,-s) \mathrm{d} s
$$

which implies

$$
K_{j}(x,-x)=\frac{\omega_{j}}{2} p(0) \mathrm{e}^{\omega_{j}^{x} j p(s) \mathrm{ds}}
$$

Similarly, we find from Equation (19) that

$$
K_{j}(x, x)=p(x) R_{j}^{+}(x)+\frac{1}{2} \int_{0}^{x} p(s) R_{j}^{+}(s) \mathrm{d} s-\omega_{j} \int_{0}^{x} p(s) K_{j}(s, s) \mathrm{d} s,
$$

that is

$$
K_{j}(x, x)=\frac{1}{2} R_{j}^{+}(x)\left(\omega_{j} p(x)+\int_{0}^{x}\left[q(s)+p^{2}(s)\right] \mathrm{d} s\right) .
$$

2) Let $x>a$. Then from integral Equations (21)-(23) we obtain the equation

$$
K_{j}\left(x,-\mu^{+}(x)\right)=\frac{\omega_{j} \alpha^{+}}{2} p(0)+\omega_{j} \int_{0}^{x} p(x, s) p(s) K_{j}\left(s,-\mu^{+}(s)\right) \mathrm{d} s .
$$


Now using 1) we easily find that

$$
K_{j}\left(x,-\mu^{+}(x)\right)=\frac{\omega_{j} \alpha^{+}}{2} p(0) \mathrm{e}^{\omega_{j} \int_{0}^{x} \frac{p(t)}{\sqrt{\rho(t)}} \mathrm{dt}} .
$$

Hence, combining the formulas (35) and (37) we obtain

$$
K_{j}\left(x,-\mu^{+}(x)\right)=\frac{\omega_{j} p(0)}{2}\left(1+\frac{1}{\sqrt{\rho(x)}}\right) \mathrm{e}^{\omega_{j} j \frac{p(t)}{0} \sqrt{\rho(t)} \mathrm{dt}} .
$$

From the integral Equations (21)-(23) it is clear that the function $K_{j}(x,) \quad.(x>a)$ has a jump discontinuity at points $\pm \mu^{-}(x)$. Computing the jumps $K_{j}\left(x, \pm \mu^{-}(x)-0\right)-K_{j}\left(x, \pm \mu^{-}(x)+0\right)$ we have

$$
K_{j}\left(x,-\mu^{-}(x)-0\right)-K_{j}\left(x,-\mu^{-}(x)+0\right)=\frac{\omega_{j} \alpha^{-} p(0)}{2} \mathrm{e}^{\omega_{j}^{j} \int_{0}^{a} p(t) d t}
$$

and

$$
\begin{aligned}
& K_{j}\left(x, \mu^{-}(x)-0\right)-K_{j}\left(x, \mu^{-}(x)+0\right) \\
& =R_{j}^{-}(x)\left[\frac{1}{2} \int_{0}^{a}\left[q(s)+p^{2}(s)\right] \mathrm{d} s-\frac{1}{2 \alpha} \int_{a}^{x}\left[q(s)+\frac{p^{2}(s)}{\alpha^{2}}\right] \mathrm{d} s+\frac{\omega_{j}}{2 \alpha^{2}} p(x)+\frac{\omega_{j}}{2}\left(1+\frac{1}{\alpha}\right)^{2} p(a)\right] .
\end{aligned}
$$

Finally, from (23) we find that

$$
K_{j}\left(x, \mu^{+}(x)\right)=R_{j}^{+}(x)\left[\frac{1}{2} \int_{0}^{a}\left[q(s)+p^{2}(s)\right] \mathrm{d} s+\frac{1}{2 \alpha} \int_{a}^{x}\left(q(s)+\frac{p^{2}(s)}{\alpha^{2}}\right) \mathrm{d} s+\frac{\omega_{j}}{2 \alpha^{2}} p(x)+\frac{\omega_{j}}{2}\left(1-\frac{1}{\alpha}\right)^{2} p(a)\right] .
$$

Hence, combining the formulas (36) and (41) we obtain

$$
K_{j}\left(x, \mu^{+}(x)\right)=\frac{1}{2} R_{j}^{+}(x)\left[\int_{0}^{x}\left(\frac{q(s)}{\sqrt{\rho(s)}}+\frac{p^{2}(s)}{\rho(s) \sqrt{\rho(s)}}\right) \mathrm{d} s+\frac{\omega_{j}}{\rho(x)} p(x)+\omega_{j}\left(1-\frac{1}{\sqrt{\rho(x)}}\right)^{2} p(a)\right] .
$$

Now we investigate the additional properties of the function $K_{j}(x,$.$) . Consider the successive approximation$ (24)-(26). By the differentiation with respect to the variable $t$ we find

$$
\begin{aligned}
D_{t} K_{j}^{(0)}(x, t) & =\frac{1}{4} q\left(\frac{x+t}{2}\right) R_{j}^{+}\left(\frac{x+t}{2}\right)+\frac{\omega_{j}}{4}\left[p^{\prime}\left(\frac{x+t}{2}\right)-\omega_{j} p^{2}\left(\frac{x+t)}{2}\right)\right] R_{j}^{+}\left(\frac{x+t}{2}\right),-x<t<x \\
D_{t} K_{j}^{(n)}(x, t)= & \frac{1}{2} \int_{\frac{x+t}{2}}^{x} q(s) K_{j}^{(n-1)}(s, t+x-s) \mathrm{d} s-\frac{1}{2} \int_{\frac{x-t}{2}}^{x} q(s) K_{j}^{(n-1)}(s, t-x+s) \mathrm{d} s \\
& -\frac{\omega_{j}}{2} p\left(\frac{x-t}{2}\right) K_{j}^{(n-1)}\left(\frac{x-t}{2},-\frac{x-t}{2}\right)-\frac{\omega_{j}}{2} p\left(\frac{x+t}{2}\right) K_{j}^{(n-1)}\left(\frac{x+t}{2}, \frac{x+t}{2}\right) \\
& -\left.\omega_{j} \int_{\frac{x-t}{2}}^{x} q(s) D_{\xi} K_{j}^{(n-1)}(s, \xi)\right|_{\xi=t-x+s} \mathrm{~d} s+\left.\omega_{j} \int_{\frac{x+t}{2}}^{x} q(s) D_{\xi} K_{j}^{(n-1)}(s, \xi)\right|_{\xi=t+x-s} \mathrm{~d} s,-x<t<x, \\
D_{t} K_{j}^{(0)}(x, t) & =\frac{\alpha^{+}}{4} q\left(\frac{\mu^{+}(x)+t}{2}\right) R_{j}^{+}\left(\frac{\mu^{+}(x)+t}{2}\right)+\frac{\omega_{j} \alpha^{+}}{4} p^{\prime}\left(\frac{\mu^{+}(x)+t}{2}\right) R_{j}^{+}\left(\frac{t+\mu^{+}(x)}{2}\right) \\
& +\frac{\alpha^{+}}{4} p^{2}\left(\frac{\mu^{+}(x)+t}{2}\right) R_{j}^{+}\left(\frac{t+\mu^{+}(x)}{2}\right),
\end{aligned}
$$




$$
\begin{aligned}
& D_{t} K_{j}^{(n)}(x, t)=\frac{\alpha^{+}}{2} \int_{\frac{\mu^{+}(x)+t}{2}}^{a} q(s) K_{j}^{(n-1)}\left(s, t+\mu^{+}(x)-s\right) \mathrm{d} s-\frac{\alpha^{-}}{2} \int_{\frac{\mu^{-}(x)-t}{2}}^{a} q(s) K_{j}^{(n-1)}\left(s, t-\mu^{-}(x)+s\right) \mathrm{d} s \\
& +\frac{1}{2 \alpha} \int_{a}^{x} q(s) K_{j}^{(n-1)}\left(s, t+\mu^{+}(x)-\mu^{+}(s)\right) \mathrm{d} s-\frac{1}{2 \alpha} \int_{a-\frac{\mu^{-}(x)+t}{2 \alpha}}^{x} q(s) K_{j}^{(n-1)}\left(s, t-\mu^{+}(x)+\mu^{+}(s)\right) \mathrm{d} s \\
& -\frac{\omega_{j} \alpha^{+}}{2} p\left(\frac{t+\mu^{+}(x)}{2}\right) K_{j}^{(n-1)}\left(\frac{t+\mu^{+}(x)}{2}, \frac{t+\mu^{+}(x)}{2}\right)+\left.\omega_{j} \alpha^{+} \int_{\frac{t+\mu^{+}(x)}{2}}^{a} p(s) D_{\xi} K_{j}^{(n-1)}(s, \xi)\right|_{\xi=t+\mu^{+}(x)-s} \mathrm{~d} s \\
& -\left.\omega_{j} \alpha^{-} \int_{\frac{\mu^{-}(x)-t}{2}}^{a} p(s) D_{\xi} K_{j}^{(n-1)}(s, \xi)\right|_{\xi=t-\mu^{-}(x)+s} \mathrm{~d} s-\frac{\omega_{j} \alpha^{-}}{2} p\left(\frac{\mu^{-}(x)-t}{2}\right) K_{j}^{(n-1)}\left(\frac{\mu^{-}(x)-t}{2},-\frac{\mu^{-}(x)-t}{2}\right) \\
& -\left.\frac{\omega_{j}}{\alpha} \int_{a-\frac{\mu^{-}(x)+t}{2 \alpha}}^{x} p(s) D_{\xi} K_{j}^{(n-1)}(s, \xi)\right|_{\xi=t-\mu^{+}(x)+\mu^{+}(s)} \mathrm{d} s+\left.\frac{\omega_{j}}{\alpha} \int_{a}^{x} p(s) D_{\xi} K_{j}^{(n-1)}(s, \xi)\right|_{\xi=t+\mu^{+}(x)-\mu^{+}(s)} \mathrm{d} s, \\
& x>a,-\boldsymbol{\mu}^{+}(x) \leq \boldsymbol{t}<-\boldsymbol{\mu}^{-}(x), n=1,2, \cdots \\
& D_{t} K_{j}^{(0)}(x, t)=\frac{\alpha^{+}}{4} q\left(\frac{\mu^{+}(x)+t}{2}\right) R_{j}^{+}\left(\frac{\mu^{+}(x)+t}{2}\right)+\frac{\alpha^{-}}{4} q\left(\frac{\mu^{-}(x)+t}{2}\right) R_{j}^{+}\left(\frac{\mu^{-}(x)+t}{2}\right) \\
& +\frac{\omega_{j} \alpha^{+}}{2}\left[p^{\prime}\left(\frac{t+\mu^{+}(x)}{2}\right)-\omega_{j} p^{2}\left(\frac{t+\mu^{+}(x)}{2}\right)\right] R_{j}^{+}\left(\frac{t+\mu^{+}(x)}{2}\right) \\
& +\frac{\omega_{j} \alpha^{-}}{2}\left[p^{\prime}\left(\frac{t+\mu^{-}(x)}{2}\right)-\omega_{j} p^{2}\left(\frac{t+\mu^{-}(x)}{2}\right)\right] R_{j}^{+}\left(\frac{t+\mu^{-}(x)}{2}\right), \\
& D_{t} K_{j}^{(n)}(x, t)=\frac{\alpha^{+}}{2} \int_{\frac{\mu^{+}(x)+t}{2}}^{a} q(s) K_{j}^{(n-1)}\left(s, t+\mu^{+}(x)-s\right) \mathrm{d} s-\frac{\alpha^{+}}{2} \int_{\frac{\mu^{+}(x)-t}{2}}^{a} q(s) K_{j}^{(n-1)}\left(s, t-\mu^{+}(x)+s\right) \mathrm{d} s \\
& -\frac{\alpha^{-}}{2} \int_{\frac{\mu^{-}(x)-t}{2}}^{a} q(s) K_{j}^{(n-1)}\left(s, t-\mu^{-}(x)+s\right) \mathrm{d} s+\frac{\alpha^{-}}{2} \int_{\frac{\mu^{-}(x)+t}{2}}^{a} q(s) K_{j}^{(n-1)}\left(s, t+\mu^{-}(x)-s\right) \mathrm{d} s \\
& +\frac{1}{2 \alpha} \int_{a}^{x} q(s)\left[K_{j}^{(n-1)}\left(s, t+\mu^{+}(x)-\mu^{+}(s)\right)-K_{j}^{(n-1)}\left(s, t-\mu^{+}(x)+\mu^{+}(s)\right)\right] \mathrm{d} s \\
& -\frac{\omega_{j} \alpha^{+}}{2} p\left(\frac{t+\mu^{+}(x)}{2}\right) K_{j}^{(n-1)}\left(\frac{t+\mu^{+}(x)}{2}, \frac{t+\mu^{+}(x)}{2}\right)+\left.\omega_{j} \alpha^{+} \int_{\frac{t+\mu^{+}(x)}{2}}^{a} p(s) D_{\xi} K_{j}^{(n-1)}(s, \xi)\right|_{\xi=t+\mu^{+}(x)-s} \mathrm{~d} s \\
& -\left.\omega_{j} \alpha^{+} \int_{\frac{\mu^{+}(x)-t}{2}}^{a} p(s) D_{\xi} K_{j}^{(n-1)}(s, \xi)\right|_{\xi=t-\mu^{+}(x)+s} \mathrm{~d} s-\left.\omega_{j} \alpha^{-} \int_{\frac{\mu^{-}(x)-t}{2}}^{a} p(s) D_{\xi} K_{j}^{(n-1)}(s, \xi)\right|_{\xi=t-\mu^{-}(x)+s} \mathrm{~d} s \\
& +\left.\omega_{j} \alpha^{-} \int_{\frac{\mu^{-}(x)+t}{2}}^{a} p(s) D_{\xi} K_{j}^{(n-1)}(s, \xi)\right|_{\xi=t+\mu^{-}(x)-s} \mathrm{~d} s-\frac{\omega_{j} \alpha^{+}}{2} p\left(\frac{\mu^{+}(x)-t}{2}\right) K_{j}^{(n-1)}\left(\frac{\mu^{+}(x)-t}{2},-\frac{\mu^{+}(x)-t}{2}\right) \\
& -\frac{\omega_{j} \alpha^{-}}{2} p\left(\frac{\mu^{-}(x)-t}{2}\right) K_{j}^{(n-1)}\left(\frac{\mu^{-}(x)-t}{2},-\frac{\mu^{-}(x)-t}{2}\right)-\left.\frac{\omega_{j}}{\alpha} \int_{a}^{x} p(s) D_{\xi} K_{j}^{(n-1)}(s, \xi)\right|_{\xi=t-\mu^{+}(x)+\mu^{+}(s)} \mathrm{d} s \\
& +\left.\frac{\omega_{j}}{\alpha} \int_{a}^{x} p(s) D_{\xi} K_{j}^{(n-1)}(s, \xi)\right|_{\xi=t+\mu^{+}(x)-\mu^{+}(s)} \mathrm{d} s, \quad x>a,-\boldsymbol{\mu}^{-}(x) \leq \boldsymbol{t}<\boldsymbol{\mu}^{-}(x), n=1,2, \cdots,
\end{aligned}
$$




$$
\begin{aligned}
& K_{j}^{(0)}(x, t)=\frac{\alpha^{-}}{4} q\left(\frac{\mu^{-}(x)+t}{2}\right) R_{j}^{+}\left(\frac{\mu^{-}(x)+t}{2}\right)+\frac{1}{4 \alpha^{2}} q\left(a+\frac{t-\mu^{-}(x)}{2 \alpha}\right) R_{j}^{+}\left(a+\frac{t-\mu^{-}(x)}{2 \alpha}\right) \\
& -\frac{1}{4 \alpha^{2}} q\left(\frac{\mu^{+}(x)-t}{2 \alpha}+a\right) R_{j}^{-}\left(\frac{\mu^{+}(x)-t}{2 \alpha}+a\right) \\
& +\frac{\omega_{j}}{4 \alpha^{3}}\left[p^{\prime}\left(\frac{t-\mu^{-}(x)}{2 \alpha}+a\right)-\frac{\omega_{j}}{\alpha} p^{2}\left(\frac{t-\mu^{-}(x)}{2 \alpha}+a\right)\right] R_{j}^{+}\left(\frac{t-\mu^{-}(x)}{2 \alpha}+a\right) \\
& +\frac{\omega_{j}}{4 \alpha^{3}}\left[p^{\prime}\left(\frac{\mu^{+}(x)-t}{2 \alpha}+a\right)+\frac{\omega_{j}}{\alpha} p^{2}\left(\frac{\mu^{+}(x)-t}{2 \alpha}+a\right)\right] R_{j}^{-}\left(\frac{\mu^{+}(x)-t}{2 \alpha}+a\right) \\
& +\frac{\omega_{j} \alpha^{-}}{4}\left[p^{\prime}\left(\frac{t+\mu^{-}(x)}{2}\right)-\omega_{j} p^{2}\left(\frac{t+\mu^{-}(x)}{2}\right)\right] R_{j}^{+}\left(\frac{t+\mu^{-}(x)}{2}\right), \\
& D_{t} K_{j}^{(n)}(x, t)=-\frac{\alpha^{+}}{2} \int_{\frac{\mu^{+}(x)-t}{2}}^{a} q(s) K_{j}^{(n-1)}\left(s, t-\mu^{+}(x)+s\right) \mathrm{d} s+\frac{\alpha^{-}}{2} \int_{\frac{\mu^{-}(x)+t}{2}}^{a} q(s) K_{j}^{(n-1)}\left(s, t+\mu^{-}(x)-s\right) \mathrm{d} s \\
& +\frac{1}{2 \alpha} \int_{a+\frac{t+\mu^{-}(x)}{2 \alpha}}^{x} q(s) K_{j}^{(n-1)}\left(s, t+\mu^{+}(x)-\mu^{+}(s)\right) \mathrm{d} s-\frac{1}{2 a} \int_{a}^{x} q(s) K_{j}^{(n-1)}\left(s, t-\mu^{+}(x)+\mu^{+}(s)\right) \mathrm{d} s \\
& -\left.\omega_{j} \alpha^{+} \int_{\frac{\mu^{+}(x)-t}{2}}^{a} p(s) D_{\xi} K_{j}^{(n-1)}(s, \xi)\right|_{\xi=t-\mu^{+}(x)+s} \mathrm{~d} s+\left.\omega_{j} \alpha^{-} \int_{\frac{\mu^{-}(x)+t}{2}}^{a} p(s) D_{\xi} K_{j}^{(n-1)}(s, \xi)\right|_{\xi=t+\mu^{-}(x)-s} \\
& -\frac{\omega_{j} \alpha^{+}}{2} p\left(\frac{\mu^{+}(x)-t}{2}\right) K_{j}^{(n-1)}\left(\frac{\mu^{+}(x)-t}{2},-\frac{\mu^{+}(x)-t}{2}\right) \\
& -\frac{\omega_{j} \alpha^{-}}{2} p\left(\frac{\mu^{-}(x)+t}{2}\right) K_{j}^{(n-1)}\left(\frac{\mu^{-}(x)+t}{2}, \frac{\mu^{-}(x)+t}{2}\right) \\
& -\frac{\omega_{j}}{2 \alpha^{2}} p\left(a+\frac{t+\mu^{-}(x)}{2 \alpha}\right) K_{j}^{(n-1)}\left(a+\frac{t+\mu^{-}(x)}{2 \alpha}, \frac{\mu^{+}(x)+t}{2}\right) \\
& -\left.\frac{\omega_{j}}{\alpha} \int_{a}^{x} p(s) D_{\xi} K_{j}^{(n-1)}(s, \xi)\right|_{\xi=t-\mu^{+}(x)+\mu^{+}(s)} \mathrm{d} s+\left.\frac{\omega_{j}}{\alpha} \int_{a+\frac{t+\mu^{-}(x)}{2 \alpha}}^{x} p(s) D_{\xi} K_{j}^{(n-1)}(s, \xi)\right|_{\xi=t+\mu^{+}(x)-\mu^{+}(s)} \mathrm{d} s, \\
& x>a, \boldsymbol{\mu}^{-}(x)<\boldsymbol{t}<\boldsymbol{\mu}^{+}(x), n=1,2, \cdots
\end{aligned}
$$

Therefore we have

$$
\begin{aligned}
& \int_{-x}^{x} D_{t}\left|K_{j}^{(0)}(x, t)\right| \mathrm{d} t \leq \frac{1}{2} \int_{0}^{x}\left[|q(s)|+\left|p^{\prime}(s)\right|+\left|p^{2}(s)\right|\right] \mathrm{d} s, 0 \leq x \leq a, \\
& \int_{-\mu^{+}(x)}^{\mu^{+}(x)}\left|D_{t} K_{j}^{(0)}(x, t)\right| \mathrm{d} t \leq \frac{\alpha^{+}+\left|\alpha^{-}\right|}{2} \int_{0}^{a}\left[|q(s)|+\left|p^{\prime}(s)\right|+\left|p^{2}(s)\right|\right] \mathrm{d} s \\
&+\left(\alpha^{+}+\left|\alpha^{-}\right|\right) \int_{a}^{x}\left[|q(s)|+\frac{\left|p^{\prime}(s)\right|}{\sqrt{\rho(s)}}+\frac{p^{2}(s)}{\rho(s)}\right] \mathrm{d} s \\
&<\left(\alpha^{+}+\left|\alpha^{-}\right|\right) \int_{0}^{x}\left[|q(s)|+\frac{\left|p^{\prime}(s)\right|}{\sqrt{\rho(s)}}+\frac{\left|p^{2}(s)\right|}{\rho(s)}\right] \mathrm{d} s, x>a .
\end{aligned}
$$




\section{Hence}

$$
\int_{-\mu^{+}(x)}^{\mu^{+}(x)}\left|D_{t} K_{j}^{(0)}(x, t)\right| \mathrm{d} t \leq C_{0} \int_{0}^{x}\left[|q(s)|+\frac{\left|p^{\prime}(s)\right|}{\sqrt{\rho(s)}}+\frac{\left|p^{2}(s)\right|}{\rho(s)}\right] \mathrm{d} s
$$

Further, because of

$$
\begin{aligned}
\int_{-x}^{x} D_{t}\left|K_{j}^{(n)}(x, t)\right| \mathrm{d} t & \int_{0}^{x}|p(s)||K(s,-s)| \mathrm{d} s+\int_{0}^{x}|p(s)|\left|K_{j}^{(n-1)}(s, s)\right| \mathrm{d} s \\
& +\int_{0}^{x}|q(s)| \mathrm{d} s \int_{-s}^{s}\left|K_{j}^{(n-1)}(s, t)\right| \mathrm{d} t \\
& +2 \int_{0}^{x}|p(s)| \mathrm{d} s \int_{-s}^{s} D_{t}\left|K_{j}^{(n-1)}(s, t)\right| \mathrm{d} t, 0 \leq x \leq a, \\
\int_{-\mu^{+}(x)}^{\mu^{+}(x)}\left|D_{t} K_{j}^{(n)}(x, t)\right| \mathrm{d} t \leq & \left(\alpha^{+}+\left|\alpha^{-}\right|\right) \int_{0}^{x}|q(s)| \mathrm{d} s \int_{-\mu^{+}(s)}^{\mu^{+}(s)}\left|K_{j}^{(n-1)}(s, t)\right| \mathrm{d} t \\
& +\left(\alpha^{+}+\left|\alpha^{-}\right|\right) \int_{0}^{x}|p(s)|\left|K_{j}^{(n-1)}\left(s,-\mu^{+}(s)\right)\right| \mathrm{d} s \\
& +\left(\alpha^{+}+\left|\alpha^{-}\right|\right) \int_{0}^{x}|p(s)|\left|K_{j}^{(n)}\left(s, \mu^{+}(s)\right)\right| \mathrm{d} s \\
& +2\left(\alpha^{+}+\left|\alpha^{-}\right|\right) \int_{0}^{x}|p(s)| \mathrm{d} s \int_{-\mu^{+}(s)}^{\mu^{+}(s)}\left|D_{t} K_{j}^{(n)}(s, t)\right| \mathrm{d} t, x>a
\end{aligned}
$$

we can write for all $x \in[0, \pi]$

$$
\begin{aligned}
\int_{-\mu^{+}(x)}^{\mu^{+}(x)}\left|D_{t} K_{j}^{(n)}(x, t)\right| \mathrm{d} t \leq & C_{0} \int_{0}^{x}|q(s)| \mathrm{d} s \int_{-\mu^{+}(s)}^{\mu^{+}(s)}\left|K_{j}^{(n-1)}(s, t)\right| \mathrm{d} t \\
& +C_{0} \int_{0}^{x}|p(s)|\left|K_{j}^{(n-1)}\left(s,-\mu^{+}(s)\right)\right| \mathrm{d} s \\
& +C_{0} \int_{0}^{x}|p(s)|\left|K_{j}^{(n-1)}\left(s, \mu^{+}(s)\right)\right| \mathrm{d} s \\
& +2 C_{0} \int_{0}^{x}|p(s)| \mathrm{d} s \int_{-\mu^{+}(s)}^{\mu^{+}(s)}\left|D_{t} K_{j}^{(n-1)}(s, t)\right| \mathrm{d} t .
\end{aligned}
$$

Note that

$$
\begin{gathered}
\int_{-\mu^{+}(x)}^{\mu^{+}(x)}\left|K_{j}^{(n-1)}(x, t)\right| \mathrm{d} t \leq \frac{\sigma^{n}(x)}{n !}, \\
\int_{0}^{x}|p(s)|\left|K_{j}^{(n-1)}\left(s, \pm \mu^{+}(s)\right)\right| \mathrm{d} s \leq A C^{n}\left(\frac{1}{n !} \int_{0}^{x}|p(s)| \mathrm{d} s\right)^{n},
\end{gathered}
$$

where $C=\max \left(\alpha^{+}, \frac{1}{\alpha}\right)$ and $A\left(0<A \leq \frac{1}{2} \int_{0}^{\pi}\left[|q(s)|+\left|p^{\prime}(s)\right|\right] \mathrm{d} s+\frac{|p(0)|}{2}\right)$ is a constant. We see that $C \leq C_{0}$ and from (44) we immediately have 


$$
\begin{aligned}
\int_{-\mu^{+}(x)}^{\mu^{+}(x)}\left|D_{t} K_{j}^{(n)}(x, t)\right| \mathrm{d} t & \leq C_{0} \int_{0}^{x}|q(s)| \frac{\sigma^{n}(s)}{n !} \mathrm{d} s+2 A C_{0} \frac{1}{n !}\left(C \int_{0}^{x}|p(t)| \mathrm{d} t\right)^{n}+2 C_{0} \int_{0}^{x}|p(s)| \mathrm{d} s \int_{-\mu^{+}(s)}^{\mu^{+}(s)}\left|D_{t} K_{j}^{(n-1)}(s, t)\right| \mathrm{d} t \\
& \leq \frac{C_{0} \sigma^{n}(x)}{n !} \int_{0}^{\pi}|q(s)| \mathrm{d} s+2 A C_{0} \frac{1}{n !}\left(2 C_{0} \int_{0}^{x}|p(t)| \mathrm{d} t\right)^{n}+2 C_{0} \int_{0}^{x}|p(s)| \mathrm{d} s \int_{-\mu^{+}(s)}^{\mu^{+}(s)}\left|D_{t} K_{j}^{(n-1)}(s, t)\right| \mathrm{d} t \\
& \leq 4 C_{0} A \frac{\sigma^{n}(x)}{n !}+2 C_{0} \int_{0}^{x}|p(s)| \mathrm{d} s \int_{-\mu^{+}(s)}^{\mu^{+}(s)}\left|D_{t} K_{j}^{(n-1)}(s, t)\right| \mathrm{d} t
\end{aligned}
$$

for all $x \in[0, \pi]$ and $n=1,2, \cdots$. Consequently,

$$
\int_{-\mu^{+}(x)}^{\mu^{+}(x)}\left|D_{t} K_{j}^{(n)}(x, t)\right| \mathrm{d} t \leq 4 C_{0} A \frac{\sigma^{n+1}(x)}{n !}+\frac{\sigma_{1}^{n+1}(x)}{(n+1) !},
$$

where $x \in[0, \pi], \quad n=0,1, \cdots$,

$$
\sigma_{1}(x)=2 C_{0} \int_{0}^{x}\left[|q(s)|+\frac{\left|p^{\prime}(s)\right|}{\sqrt{\rho(s)}}+\frac{\left|p^{2}(s)\right|}{\rho(s)}+|p(s)|\right] \mathrm{d} s .
$$

This means that the series

$$
\sum_{n=0}^{\infty} K_{j}^{(n)}(x, .)
$$

can be differentiated term by term in the space $L_{1}\left(-\mu^{+}(x), \mu^{+}(x)\right)$ and the sum $K_{j}(x,$.$) is also differentia-$ ble in this space with

$$
\int_{-\mu^{+}(x)}^{\mu^{+}(x)}\left|D_{t} K_{j}(x, t)\right| \mathrm{d} t \leq 4 C_{0} A \sigma(x) \mathrm{e}^{\sigma(x)}+\mathrm{e}^{\sigma_{1}(x)}-1
$$

Similarly, from the successive approximation (24)-(26) by differentiation with respect to the variable $x$ we have the series

$$
\sum_{n=0}^{\infty} D_{x} K_{j}^{(n)}(x, .)
$$

converges in the space $L_{1}\left(-\mu^{+}(x), \mu^{+}(x)\right)$ and $D_{x} K_{j}(x,.) \in L_{1}\left(-\mu^{+}(x), \mu^{+}(x)\right)$.

Further,by differentiation integral Equations (19), (21)-(23) we have that

$$
\begin{aligned}
D_{t} K_{j}(x, t)-D_{x} K_{j}(x, t)= & -\frac{\omega_{j}}{2} p\left(\frac{x-t}{2}\right) K_{j}\left(\frac{x-t}{2},-\frac{x-t}{2}\right)-\int_{\frac{x-t}{2}}^{x} q(s) K_{j}(s, t-x+s) \mathrm{d} s \\
& -\left.\omega_{j} \int_{\frac{x-t}{2}}^{x} q(s) D_{\xi} K_{j}(s, \xi)\right|_{\xi=t-x+s} \mathrm{~d} s,-x<t<x, 0 \leq x \leq a, \\
\alpha D_{t} K_{j}(x, t)-D_{x} K_{j}(x, t)= & -\int_{a-\frac{\mu^{-}(x)+t}{2 \alpha}}^{x} q(s) K_{j}\left(s, t-\mu^{+}(x)+\mu^{+}(s)\right) \mathrm{d} s \\
& -\left.2 \omega_{j} \int_{a-\frac{\mu^{-}(x)+t}{2 \alpha}}^{x} p(s) D_{\xi} K_{j}^{(n-1)}(s, \xi)\right|_{\xi=t-\mu^{+}(x)+\mu^{+}(s)} \mathrm{d} s \\
& -\frac{\omega_{j}}{\alpha} p\left(a-\frac{\mu^{-}(x)+t}{2}\right) K_{j}\left(a-\frac{\mu^{-}(x)+t}{2},-\frac{\mu^{+}(x)-t}{2}\right), \\
& x>a,-\mu^{+}(x) \leq \boldsymbol{t}<-\mu^{-}(x),
\end{aligned}
$$




$$
\begin{aligned}
& \alpha D_{t} K_{j}(x, t)-D_{x} K_{j}(x, t) \\
& =\frac{\alpha \alpha^{-}}{2} q\left(\frac{\mu^{-}(x)+t}{2}\right) R_{j}^{+}\left(\frac{\mu^{-}(x)+t}{2}\right)+\left[\frac{\omega_{j} \alpha \alpha^{-}}{2} p^{\prime}\left(\frac{t+\mu^{-}(x)}{2}\right)-\omega_{j} p^{2}\left(\frac{t+\mu^{-}(x)}{2}\right)\right] R_{j}^{+}\left(\frac{t+\mu^{-}(x)}{2}\right) \\
& -\alpha \alpha^{+} \int_{\frac{\mu^{+}(x)-t}{2}}^{a} q(s) K_{j}\left(s, t-\mu^{+}(x)+s\right) \mathrm{d} s+\alpha \alpha^{-} \int_{\frac{\mu^{-}(x)+t}{2}}^{a} q(s) K_{j}\left(s, t+\mu^{-}(x)-s\right) \mathrm{d} s \\
& -\int_{a}^{x} q(s) K_{j}\left(s, t-\mu^{+}(x)+\mu^{+}(s)\right) \mathrm{d} s-\left.2 \omega_{j} \alpha \alpha^{+} \int_{\frac{\mu^{+}(x)-t}{2}}^{a} p(s) D_{\xi} K_{j}(s, \xi)\right|_{\xi=t-\mu^{+}(x)+s} \mathrm{~d} s \\
& +\left.2 \omega_{j} \alpha \alpha^{-} \int_{\frac{\mu^{-}(x)+t}{2}}^{a} p(s) D_{\xi} K_{j}(s, \xi)\right|_{\xi=t+\mu^{-}(x)-s}-\left.2 \omega_{j} \int_{a}^{x} p(s) D_{\xi} K_{j}^{(n-1)}(s, \xi)\right|_{\xi=t-\mu^{+}(x)+\mu^{+}(s)} \mathrm{d} s \\
& -\frac{\omega_{j} \alpha \alpha^{+}}{2} p\left(\frac{\mu^{+}(x)-t}{2}\right) K_{j}\left(\frac{\mu^{+}(x)-t}{2},-\frac{\mu^{+}(x)-t}{2}\right) \\
& -\frac{\omega_{j} \alpha \alpha^{-}}{2} p\left(\frac{\mu^{-}(x)+t}{2}\right) K_{j}\left(\frac{\mu^{-}(x)+t}{2}, \frac{\mu^{-}(x)+t}{2}\right), x>a,-\mu^{-}(x) \leq \boldsymbol{t}<\mu^{-}(x), \\
& \alpha D_{t} K_{j}(x, t)-D_{x} K_{j}(x, t) \\
& =\frac{\alpha \alpha^{-}}{4} q\left(\frac{\mu^{-}(x)+t}{2}\right) R_{j}^{+}\left(\frac{\mu^{-}(x)+t}{2}\right)-\frac{1}{2 \alpha} q\left(\frac{\mu^{+}(x)-t}{2 \alpha}+a\right) R_{j}^{-}\left(\frac{\mu^{+}(x)-t}{2 \alpha}+a\right) \\
& +\frac{\omega_{j}}{2 \alpha^{2}}\left[p^{\prime}\left(\frac{\mu^{+}(x)-t}{2 \alpha}+a\right)+\frac{\omega_{j}}{\alpha} p^{2}\left(\frac{\mu^{+}(x)-t}{2 \alpha}+a\right)\right] R_{j}^{-}\left(\frac{\mu^{+}(x)-t}{2 \alpha}+a\right) \\
& +\frac{\omega_{j} \alpha \alpha^{-}}{2}\left[p^{\prime}\left(\frac{t+\mu^{-}(x)}{2}\right)-\omega_{j} p^{2}\left(\frac{t+\mu^{-}(x)}{2}\right)\right] R_{j}^{+}\left(\frac{t+\mu^{-}(x)}{2}\right) \\
& -\alpha \alpha^{+} \int_{\frac{\mu^{+}(x)-t}{2}}^{a} q(s) K_{j}\left(s, t-\mu^{+}(x)+s\right) \mathrm{d} s+\alpha \alpha^{-} \int_{\frac{\mu^{-}(x)+t}{2}}^{a} q(s) K_{j}\left(s, t+\mu^{-}(x)-s\right) \mathrm{d} s \\
& -\int_{a}^{x} q(s) K_{j}\left(s, t-\mu^{+}(x)+\mu^{+}(s)\right) \mathrm{d} s-\left.2 \omega_{j} \alpha \alpha^{+} \int_{\frac{\mu^{+}(x)-t}{2}}^{a} p(s) D_{\xi} K_{j}(s, \xi)\right|_{\xi=t-\mu^{+}(x)+s} \mathrm{~d} s \\
& +\left.2 \omega_{j} \alpha \alpha^{-} \int_{\frac{\mu^{-}(x)+t}{2}}^{a} p(s) D_{\xi} K_{j}(s, \xi)\right|_{\xi=t+\mu^{-}(x)-s}-\left.2 \omega_{j} \int_{a}^{x} p(s) D_{\xi} K_{j}(s, \xi)\right|_{\xi=t-\mu^{+}(x \mathrm{ds})+\mu^{+}(s)} \\
& -\omega_{j} \alpha \alpha^{+} p\left(\frac{\mu^{+}(x)-t}{2}\right) K_{j}\left(\frac{\mu^{+}(x)-t}{2},-\frac{\mu^{+}(x)-t}{2}\right)-\omega_{j} \alpha \alpha^{-} p\left(\frac{\mu^{-}(x)+t}{2}\right) K_{j}\left(\frac{\mu^{-}(x)+t}{2}, \frac{\mu^{-}(x)+t}{2}\right), \\
& x>a, \boldsymbol{\mu}^{-}(x)<\boldsymbol{t}<\boldsymbol{\mu}^{+}(x) .
\end{aligned}
$$

These equations with (47) imply that

$$
\int_{-\mu^{+}(x)}^{\mu^{+}(x)}\left|D_{x} K_{j}(x, t)\right| \mathrm{d} t \leq 4 \sqrt{\rho(x)} C_{0} A \sigma(x) \mathrm{e}^{\sigma(x)}+\sqrt{\rho(x)}\left(\mathrm{e}^{\sigma_{1}(x)}-1\right)+C_{1} \sigma_{1}(x),
$$

where $C_{1}>0$ is a constant. Differentiating Equations (48)-(51) once more we have the following partial differential equation for the kernel $K_{j}(x, t)$ : 


$$
D_{x x} K_{j}(x, t)-\rho(x) D_{t t} K_{j}(x, t)=q(x) K_{j}(x, t)+2 \omega_{j} p(x) D_{t} K_{j}(x, t) .
$$

Hence we can formulate the following theorem:

Theorem 2 For all fixed $x \in[0, \pi]$ the kernel of the integral representation (34) has the partial derivatives $D_{x} K_{j}(x,),. \quad D_{t} K_{j}(x,.) \in L_{1}\left(-\mu^{+}(x), \mu^{+}(x)\right)$ and satisfy the discontinuous partial differential Equation (53) with the conditions

$$
\begin{gathered}
K_{j}\left(x,-\mu^{+}(x)\right)=\frac{\omega_{j} p(0)}{2}\left(1+\frac{1}{\sqrt{\rho(x)}}\right) \mathrm{e}^{\omega_{j} j} \frac{p(t)}{0 \sqrt{\rho(t)}} \mathrm{dt} \\
K_{j}\left(x, \mu^{+}(x)\right)=\frac{1}{2} R_{j}^{+}(x)\left[\int_{0}^{x}\left(\frac{q(s)}{\sqrt{\rho(s)}}+\frac{p^{2}(s)}{\rho(s) \sqrt{\rho(s)}}\right) \mathrm{d} s+\frac{\omega_{j}}{\rho(x)} p(x)+\omega_{j}\left(1-\frac{1}{\sqrt{\rho(x)}}\right)^{2} p(a)\right] .
\end{gathered}
$$

and the discontinuity conditions

$$
K_{j}\left(x,-\mu^{-}(x)-0\right)-K_{j}\left(x,-\mu^{-}(x)+0\right)=\frac{\omega_{j} p(0)}{2}\left(1-\frac{1}{\sqrt{\rho(x)}}\right) \mathrm{e}^{\substack{\omega_{j} j p(t) d t \\ j}}
$$

and

$$
\begin{aligned}
& K_{j}\left(x, \mu^{-}(x)-0\right)-K_{j}\left(x, \mu^{-}(x)+0\right) \\
& =R_{j}^{-}(x)\left[\frac{1}{2} \int_{0}^{a}\left[q(s)+p^{2}(s)\right] \mathrm{d} s-\frac{1}{2 \alpha} \int_{a}^{x}\left[q(s)+\frac{p^{2}(s)}{\alpha^{2}}\right] \mathrm{d} s+\frac{\omega_{j}}{2 \alpha^{2}} p(x)+\frac{\omega_{j}}{2}\left(1+\frac{1}{\alpha}\right)^{2} p(a)\right],
\end{aligned}
$$

where

$$
R_{j}^{ \pm}(x)=r^{ \pm}(x) \mathrm{e}^{\mp \omega_{j} j \operatorname{sgn}(t \pm a) \frac{p(t)}{\sqrt{\rho(t)}} \mathrm{dt}}
$$

\section{References}

[1] McHugh, J. (1970) An Historical Survey of Ordinary Linear Differential Equations with a Large Parameter and Turning Points. Archive for History of Exact Sciences, 7, 277-324.

[2] Kostyuchenko, A.G. and Shkalikov, A.A. (1983) Selfadjoint Quadratic Operator Pencils and Elliptic Problem. Functional Analysis and Its Applications, 17, 109-128. http://dx.doi.org/10.1007/BF01083136

[3] Freiling, G. (1988) On the Completeness and Minimality of the Derived Chains of Eigen and Associated Functions of Boundary Eigenvalue Problems Nonlinearly Dependent on the Parameter. Results in Mathematics, 1464-1483.

[4] Kaup, D.J. (1975) A Higher-Order Water-Wave Equation and the Method for Solving it. Progress of Theoretical Physics, 54, 396-408. http://dx.doi.org/10.1143/PTP.54.396

[5] Cornille, H. (1970) Existence and Uniqueness of Crossing Symmetric N/D-Type Equations Corresponding to the Klein-Gordon Equation. Journal of Mathematical Physics, 11, 79-98. http://dx.doi.org/10.1063/1.1665074

[6] Weiss, R. and Scharf, G. (1971) The Inverse Problem of Potential Scattering According to the Klein-Gordon Equation. Helvetica Physica Acta, 44, 910-929.

[7] Jaulent, M. and Jean, C. (1976) The Inverse Problem for the One-Dimensional Schrödinger Equation with an EnergyDependent Potential I, II. Ann. Inst. H. Poincare Sect. A (N.S.), 25, 105-118, 119-137.

[8] Jaulent, M. (1972) On an Inverse Scattering Problem with an Energy-Dependent Potential. Ann. Inst. H. Poincare Sect. A (N.S.), 17, 363-378.

[9] Marchenko, V.A. (1977) Sturm-Liouville Operators and Their Applications. Naukova Dumka, Kiev English Trans Birkhauser, Basel.

[10] Chadan, K. and Sabatier, P.C. (1989) Inverse Problems in Quantum Scattering Theory, 2nd Edition, Springer-Verlag, New York. http://dx.doi.org/10.1007/978-3-642-83317-5

[11] Levitan, B.M. (1987) İnverse Surm-Liouville problems. VNU Science Press, Utrecht. 
[12] Levitan, B.M. and Gasymov, M.G. (1964) Determination of a Differential Equation by Two Spectra. Uspekhi Matematicheskikh Nauk, 192, 3-63. http://dx.doi.org/10.1070/rm1964v019n02abeh001145

[13] Pöschel, J. and Trubowitz, E. (1987) İnverse Spectral Theory. Academic Press, New York.

[14] Deift, P. and Trubowitz, E. (1979) Inverse Scattering on the Line. Communications on Pure and Applied Mathematics, 32, 121-251. http://dx.doi.org/10.1002/cpa.3160320202

[15] Freiling, G. and Yurko, V. (2001) Inverse Sturm-Liouville Problems and Their Applications. Nova Science Publishers, Inc., Huntington.

[16] McLaughlin, J.R. (1986) Analytical Methods for Recovering Coefficients in Differential Equations from Spectral Data. SIAM Review, 28, 53-57. http://dx.doi.org/10.1137/1028003

[17] Gasymov, M.G. and Guseinov, G.S. (1981) Determination of a Diffusion Operator from Spectral Data. Akademiya Nauk Azerbaijani SSR Doklady, 37, 19-23.

[18] Guseinov, G.S. (1985) On the Spectral Analysis of a Quadratic Pencil of Sturm-Liouville Operators. Doklady Akademii nauk SSSR, 285, 1292-1296.

[19] Guseinov, I.M. and Nabiev, I.M. (2007) An Inverse Spectral Problem for Pencils of Differential Operators. Matematicheskii Sbornik, 198, 47-66. http://dx.doi.org/10.1070/sm2007v198n11abeh003897

[20] Nabiev, I.M. (2004) The Inverse Spectral Problem for the Diffusion Operator on an Interval. Matematicheskaya Fizika, Analiz, Geometriya, 11, 302-313.

[21] Yurko, V.A. (2000) An Inverse Problem for Pencils of Differential Operators. Sbornik: Mathematics, 191, $1561-1586$. http://dx.doi.org/10.1070/SM2000v191n10ABEH000520

[22] Hryniv, R. and Pronska, N. (2012) Inverse Spectral Problem for Energy-Dependent Sturm-Liouville Equation. Inverse Problems, 28, Article ID: 085008. http://dx.doi.org/10.1088/0266-5611/28/8/085008

[23] Pronska, N. (2013) Reconstruction of Energy-Dependent Sturm-Liouville Operators from Two Spectra. Integral Equations and Operator Theory, 76, 403-419.

[24] Sattinger, D.H. and Szmigielski, J. (1995) Energy Dependent Scattering Theory. Differential Integral Equations, 8, 945-959.

[25] Tsutsumi, M. (1981) On the Inverse Scattering Problem for the One-Dimensional Schrödinger Equation with an Energy Dependent Potential. Journal of Mathematical Analysis and Applications, 83, 316-350. http://dx.doi.org/10.1016/0022-247X(81)90266-3

[26] van der Mee, C. and Pivovarchik, V. (2001) Inverse Scattering for a Schrodinger Equation with Energy Dependent Potential. Journal of Mathematical Physics, 42, 158-181. http://dx.doi.org/10.1063/1.1326921

[27] Aktosun, T. and van der Mee, C. (1991) Scattering and Inverse Scattering for the1-D Schrödinger Equation with Energy-Dependent Potentials. Journal of Mathematical Physics, 32, 2786-2801. http://dx.doi.org/10.1063/1.529070

[28] Nabiev, A.A. (2006) Inverse Scattering Problem for the Schrödinger-Type Equation with a Polynomial EnergyDependent Potential. Inverse Problems, 22, 2055-2068. http://dx.doi.org/10.1088/0266-5611/22/6/009

[29] Nabiev, A.A. and Guseinov, I.M. (2006) On the Jost Solutions of the Schrödinger-Type Equations with a Polynomial Energy-Dependent Potential. Inverse Problems, 22, 55-67. http://dx.doi.org/10.1088/0266-5611/22/1/004

[30] Kamimura, Y. (2007) An Inversion Formula in Energy Dependent Scattering. Journal of Integral Equations and Applications, 19, 473-512. http://dx.doi.org/10.1216/jiea/1192628620

[31] Yurko, V. (2006) Inverse Spectral Problems for Differential Pencils on the Half-Line with Turning Points. Journal of Mathematical Analysis and Applications, 320, 439-463. http://dx.doi.org/10.1016/j.jmaa.2005.06.085

[32] Hald, O. (1984) Discontinuous Inverse Eigenvalue Problems. Communications on Pure and Applied Mathematics, 37, 539-577. http://dx.doi.org/10.1002/cpa.3160370502

[33] Carlson, R. (1994) An Inverse Spectral Problem for Sturm-Liouville Operators with Discontinuous Coefficients. Proceedings of the American Mathematical Society, 120, 475-484. http://dx.doi.org/10.1090/S0002-9939-1994-1197532-5

[34] Yurko, V. (2000) Integral Transforms Connected with Discontinuous Boundary Value Problems. Integral Transforms and Special Functions, 10, 141-164. http://dx.doi.org/10.1080/10652460008819282

[35] Yurko, V.A. (2000) On Boundary Value Problems with Discontinuity Conditions inside an Interval. Differential Equations, 36, 1266-1269. http://dx.doi.org/10.1007/BF02754199

[36] Amirov, R.K. (2006) On Sturm-Liouville Operators with Discontinuity Conditions inside an Interval. Journal of Mathematical Analysis and Applications, 317, 163-176. http://dx.doi.org/10.1016/j.jmaa.2005.11.042

[37] Kobayashi, M. (1989) A Uniqueness Proof for Discontinuous Inverse Sturm-Liouville Problems with Symmetric Potentials. Inverse Problems, 5, 767-781. http://dx.doi.org/10.1088/0266-5611/5/5/007

[38] Guseinov, I.M. and Pashaev, R.T. (2002) On an Inverse Problem for a Second-Order Differential Equation. Russian 
Mathematical Surveys, 57, 597-598. http://dx.doi.org/10.1070/RM2002v057n03ABEH000517

[39] Akhmedova, E.N. (2002) On Representation of Solution of Sturm-Liouville Equation with Discontinuous Coefficients. Proceedings of the Institute of Mathematics and Mechanics National Academy of Sciences of Azerbaijan, 16, 208.

[40] Akhmedova, E. and Huseynov, H.M. (2003) On Eigenvalues and Eigenfunctions of One Class of Sturm-Liouville Operators with Discontinuous Coefficients. Transactions of National Academy of Sciences of Azerbaijan. Series of Physical-Technical and Mathematical Sciences, 23, 7-18.

[41] Mamedov, K.R. (2010) On an Inverse Scattering Problem for a Discontinuous Sturm-Liouville Equation with a Spectral Parameter in the Boundary Condition, Boundary Value Problems, 2010, Article ID: 171967. http://dx.doi.org/10.1155/2010/171967

[42] Nabiev Adiloglu, A. and Amirov, R.K. (2013) On the Boundary Value Problem for the Sturm-Liouville Equation with the Discontinuous Coefficient. Mathematical Methods in the Applied Sciences, 36, 1685-1700. http://dx.doi.org/10.1002/mma.2714 